\title{
Epidemiological Surveillance of Leprosy in Cameroon Using the ELISA Test with D-BSA and Tris-HCl as Buffer
}

\author{
Dickson Shey Nsagha ${ }^{1,2, *}$, Elijah Afolabi Bamgboye ${ }^{2}$, Lekan Salimonu ${ }^{3}$, Jules Clement Nguedia Assob ${ }^{4}$, Earnest \\ Njih Tabah $^{5}$, Anne-Cécile Bissek ${ }^{6}$, Anna Longdoh Njunda ${ }^{7}$, Henri-Lucien Kamga ${ }^{7}$, Marcelin Ngowe Ngowe ${ }^{8}$, Alfred \\ Kongnyu Njamnshi ${ }^{6}$

\begin{abstract}
${ }^{1}$ Department of Public Health and Hygiene, Medicine Programme, Faculty of Health Sciences, University of Buea, Buea, Cameroon ${ }^{2}$ Department of Epidemiology, Medical Statistics and Environmental Health (Formerly Department of Preventive and Social Medicine), Faculty of Public Health, College of Medicine, University of Ibadan, Ibadan, Nigeria

${ }^{3}$ Department of Chemical Pathology, Faculty of Basic Medical Sciences, College of Medicine, University of Ibadan, Ibadan, Nigeria ${ }^{4}$ Department of Biomedical Sciences, Faculty of Health Sciences, University of Buea, Buea, Cameroon

${ }^{5}$ National Programme for Leprosy, Buruli Ulcer, Leshimanisis \& Yaws Control, Ministry of Public Health, Yaounde, Cameroon ${ }^{6}$ Department of Internal Medicine \& Specialties (Dermatology and Neurology), Faculty of Medicine \& Biomedical Sciences, University of Yaounde I, Yaounde, Cameroon

${ }^{7}$ Department of Medical Laboratory Sciences, Faculty of Health Sciences, University of Buea, Buea, Cameroon ${ }^{8}$ Department of Surgery, Obstetrics and Gynaecology, Faculty of Health Sciences, University of Buea, Buea, Cameroon *Corresponding author: nsaghads@hotmail.com; dsnsagha@gmail.com
\end{abstract}

Received March 18, 2014; Revised May 04, 2014; Accepted May 04, 2014

\begin{abstract}
Background: Early detection of leprosy is important for elimination, but most cases are diagnosed only when lesions occur and nerves and skin are damaged. The determination of the risk of contacts to develop leprosy is still unpredictable, and elimination is not possible as long as contacts can still develop the disease. Methods: A modified PGL-I ELISA using tris-HCl as buffer and D-BSA as antigen evaluated IgM leprosy antibodies for the detection of subclinical leprosy infection and monitor effective chemotherapy. This case-control study took place in a leprosy endemic area among lepers diagnosed clinically and bacteriologically, intra-familial and extra-familial contacts and controls consisting of patients attending a health facility for reasons other than leprosy. Results: The highest mean absorbance of positive sera was from leprosy patients $(0.14 \pm 0.06)$, contacts $(0.12 \pm 0.05)$ and controls $(0.04 \pm 0.04)$. The proportion of sero-positive subjects for IgM anti-bodies to M. leprae was $43.5 \%$ (95\% CI: $37.6 \%$ 49.4\%). Twenty-three (44.2\%) discharged cases since 1985 were still sero-positive for leprosy IgM anti-bodies; 15 years after treatment. We found a risk factor of 6 among contacts and controls suggesting a high level of transmission. Boyo division had a higher seropositivity (51.5\%) compared to Mezam division (30\%). Positive cases were clustered among students (44.8\%), farmers (42.5\%), the self-employed (44.7\%), those with paid employment (37.5\%) and the unemployed (60.0\%) $(\mathrm{P}=0.01)$. Conclusion: The PGL-I ELISA with D-BSA and tris-HCl as buffer is a useful epidemiological surveillance tool for the detection of sub-clinical leprosy infection and chemotherapy monitoring. The discharged patients who tested positive should be re-evaluated for relapses.
\end{abstract}

Keywords: leprosy, PGL-I ELISA, D-BSA, tris - HCl, surveillance, epidemiology, Cameroon

Cite This Article: Dickson Shey Nsagha, Elijah Afolabi Bamgboye, Lekan Salimonu, Jules Clement Nguedia Assob, Earnest Njih Tabah, Anne-Cécile Bissek, Anna Longdoh Njunda, Henri-Lucien Kamga, Marcelin Ngowe Ngowe, and Alfred Kongnyu Njamnshi, "Epidemiological Surveillance of Leprosy in Cameroon Using the ELISA Test with D-BSA and Tris-HCl as Buffer." American Journal of Epidemiology and Infectious Disease, vol. 2, no. 3 (2014): 66-73. doi: 10.12691/ajeid-2-3-1.

\section{Introduction}

Early detection of leprosy is a priority for achieving control and elimination. However, clinical examination is not always possible and the majority of cases are diagnosed only when lesions occur and the nerves and skin are already damaged $[1,2]$. The determination of the risk of an infected contact to develop leprosy is still unpredictable, and leprosy elimination is not possible as long as infected contacts can still develop the disease [3]. The current leprosy control measures in Cameroon include early case detection and treatment with multi-drug therapy. While this is a sound concept for surveillance, leprosy has a long latency period, and several years of follow-up are necessary to detect early stages of the disease [4]. The "final push" by WHO to eliminate leprosy is based not only on prevalence and incidence indicators but on transmission in high risk groups such as contacts $[5,6,7]$. 
Although leprosy has been eliminated as a public health problem in Cameroon, there are still areas with high prevalence such as Essimbiland and Mbingo in the North West Region [8,9]. In the traditional PGL-1ELISA method, di-saccharide-bovine serum albumin (D-BSA) and bovine serum albumin (BSA) are used as antigen and control respectively while phosphate buffer saline is used as buffer [10]. The ELISA test was based on a synthetic conjugate containing the carbohydrate epitope of PGL-1 [11]; the major antigenic glycolipid in the bacterium. The principal antigenic determinant of PGL-1 is the di- and trisaccharide of the molecule [12]. The ELISA for PGL-1 derivatives involved the immobilisation of the protein part of the molecule (bovine saline albumine or BSA) to a plastic surface via passive interactions [12]. The test employed anti-human-IgM alkaline phosphatase as enzyme.According to the WHO, leprosy surveillance systems are an essential element in monitoring the progress being achieved towards reaching elimination [13]. These surveillance systems include monitoring and evaluation, organization of annual reporting from endemic countries to WHO and feed-back, and regular publication of progress based on essential indicators of elimination among others [13]. Active surveillance of leprosy through active case finding among extra-familial contacts is a major component of leprosy elimination [14]. The examination of household contacts of known cases has been used as a tool to increase the early diagnosis of the disease and to interrupt transmission $[15,16]$ but the utility of examination of other groups, such as neighbourhood and social contacts, is less clear [14]. In this study, a modified PGL-1 ELISA using tris- $\mathrm{HCl}$ as buffer and DBSA as antigen and BSA as control were used to provide more information as an epidemiological surveillance tool for the detection of sub-clinical leprosy infection and to monitor treatment by measuring IgM antibodies against leprosy among patients, their contacts and a control group from an endemic region.

\section{Materials and Methods}

\subsection{Study Area and Subjects}

The study took place in the former North West Province (now North West Region) of Cameroon from 1998- 2002 where leprosy is still endemic in some localities [8,9]. Specifically, patients and contacts (intrafamilial and extra-familial) were recruited from the Fundong Health District in Boyo division, where one of the oldest leprosarium in Cameroon is located at the Mbingo Baptist Hospital. The controls were recruited from the Bamenda Health District in Mezam Division which is a low endmic area for leprosy.

The sixty-four (64) consecutive leprosy patients who were diagnosed clinically based on body lesions and bacteriologically based on the presence of acid-fast bacilli/bacterial index in body lesions were used. All patients in the Mbingo leprosarium of Boyo Division and surrounding communities of Mbingo II, Mejang, Baingo, Dr Jones' quarter and NewHope village who gave a written consent, were recruited for the study.

One hundred and seven intra-familial contacts (wives, children and other relations) and extra-familial (friends, peers, colleagues and other villagers) contacts were recruited as a first group of controls from Boyo Division. A second group of 100 controls were drawn from Mezam Division which has a low prevalence of leprosy and included those who attended hospital for reasons other than leprosy; those who indicated that they had lived or were living with a leprosy patient in the same household or quarter were eliminated from the study. The leprosy patients and their contacts were purposively chosen while the controls were volunteers.

Five $\mathrm{ml}$ of venous blood was collected from each leprosy patient, contact and control subjects after disinfection with cotton wool imbibed in alcohol through a sterile syringe by the lead author. The blood samples were transferred in labelled plain bottles placed in ice bags to either the Mbingo Baptist Hospital or Bamenda Regional Hospital. The blood samples collected were centrifuged after 30minutes of collection and the serum was separated and preserved in labelled aliquot tubes in a freezer. The serum samples were finally transported in ice bags to the University College Hospital, Ibadan, Nigeria for laboratory analysis.

\subsection{Laboratory Procedures}

Carbonate-bicarbonate coating buffer [pH 9.6], citric acid phosphate buffer [pH 5], $30 \% \mathrm{H}_{2} \mathrm{O}_{2}$, tris- $\mathrm{HCl}$ buffer [pH7.4] and $3.5 \mathrm{M} \mathrm{NaCl}$ were prepared according to standard laboratory procedures. Normal goat serum (NGS) was prepared by collecting from slaughtered animals; after retraction, the blood was centrifuged at 600rpm for five minutes and the serum separated and preserved in a freezer at $-20^{\circ} \mathrm{C}$ until used. Tris- $\mathrm{HCl}$ wash solution was prepared with $100 \mathrm{ml}$ of tris- $\mathrm{HCl}$ stock plus $100 \mathrm{ml}$ 3.5MNaCl and $1 \mathrm{ml}$ Tween-20 were added together and water was added to the $1000 \mathrm{ml}$ mark. Serum dilution solution was carried out in tris- $\mathrm{HCl}-1 \% \mathrm{NGS}$.

Disaccharide-bovine serum albumin (D-BSA) and bovine serum albumin (BSA) (donated by WHOcouretesy of Dr. U. Fluth of the vaccine unit) were used as antigen and control respectively. Three $\mathrm{ml}$ of distilled water was added to each vial of lyophilised substance and unused reconstituted D-BSA and BSA were kept frozen at $-20^{\circ} \mathrm{C}$. D-BSA and BSA were diluted 1: 100 by adding $0.1 \mathrm{ml}$ of the solution to $9.9 \mathrm{ml}$ of the buffer.

\subsubsection{The PGL-1 TRIS-HCL ELISA}

Microtiter plates were coated separately with D-BSA or BSA diluted 1:100 in carbonate-carbonate buffer and the plates incubated overnight at $4^{\circ} \mathrm{C}$ for proper coating. The plates were washed three times and blocked with $50 \mu \mathrm{l}$ BSA in tris- $\mathrm{HCl}$ and diluted 1:100 and incubated for one hour at room temperature and subsequently washed three times with the wash solution.

Dilutions of the sera were carried out $1: 200 \mathrm{ml}$ in tris$\mathrm{HCl}-1 \%$ NGS. One hundred $\mu \mathrm{l}$ of each serum sample was added to the wells and incubated at $37^{\circ} \mathrm{C}$ for one hour in an incubator. After washing the wells three times, $50 \mu \mathrm{l}$ antihuman IgM peroxidase (Behring, Germany) diluted 1:500 in tris- $\mathrm{HCl}-1 \% \mathrm{NGS}$ were added to the wells and incubated at $37^{\circ} \mathrm{C}$ for one hour. The wells were washed three times and $100 \mu \mathrm{l}$ of a mixture of one tablet of orthophenylene-diamine dissolved in $10 \mathrm{ml} 0.1 \mathrm{M}$ citric acid 
(0.035mole) phosphate buffer (0.067 mole (pH 5) and $5 \mu \mathrm{l}$ $30 \% \mathrm{H} \mathrm{H}_{2} \mathrm{O}_{2}$ was added.

The plates were incubated for 15 minutes in the dark at room temperature for the colour to develop and then stopped by adding $100 \mu \mathrm{l} 2 \mathrm{~N} \mathrm{H}_{2} \mathrm{SO}_{4}$ to each well. Optical density was read at $490 \mathrm{~nm}$ using an ELISA reader (Titertek, Multiskan, Flow Laboratories, Sweden).

Each test was performed in duplicates (with D-BSA and BSA) and the mean absorbance of the wells with BSA was subtracted from one of the wells with D-BSA. Six wells in each plate were allocated for randomly chosen controls from Mezam Division. A sample was considered positive if it yielded a net absorbance greater than the total derived by adding two standard deviations (SD) to the mean $(>0+2 \mathrm{SD})$ of the absorbance for a group of the six controls. The ELISA test was carried out in four batches containing 70, 45, 78 and 78 serum samples respectively. The cut-off value was determined for each run in each plate. The cut-off value of the optical density of each batch of the ELISA test varied from 0.107 to 0.017 .

\subsection{Ethical Clearance}

The authorisation to carry out the study was obtained from the Cameroonian Ministry of Public Health $\left(\mathrm{N}^{\circ}\right.$ D76/A/MSP/SESP/SG/DRH/SDGP/SFS) who gave the ethical approval. The purposes of the study and its advantages to the participants were explained and written informed consent sought and obtained before blood samples were collected. For literate participants, they were given the consent form to read and ask questions when necessary and all questions answered to their satisfaction before they signed. For illiterate participants, an independent literate person witnessed the accurate reading of the consent form to the potential participant, and the individual had the opportunity to ask questions before thumb printing to confirm the consent.

\subsection{Data Management and Analysis}

All demographic, clinical and laboratory results were registered on structured forms that were cross-checked for correct and complete filling. Consistency checks were also conducted as well as the use of correct codes. All data were stored safely at the College of Medicine of the University of Ibadan in Nigeria. The data was analysed using Epi-Info version 6 after a double entry by two data clerks. At the initial step of the data analysis, frequency distributions of all variables were produced according to the batch of the ELISA test performed. Associations were established between variables of different measures through cross-tabulations. Further analysis included data summary such as proportions, percentages, ratios and standard deviation. In these analyses, such methods as the Chi-square test, Fisher exact test for the test of significance of associations between categorical variables and "student-t test" and ANOVA to test statistical significance of continuous variables were used.

\section{Results}

The socio-demographic characteristics of the subjects involved in this survey are described elsewhere [9]. Briefly, participants included men and women of different age groups, marital and social status, whether they had received the BCG vaccine, educational level and religion.

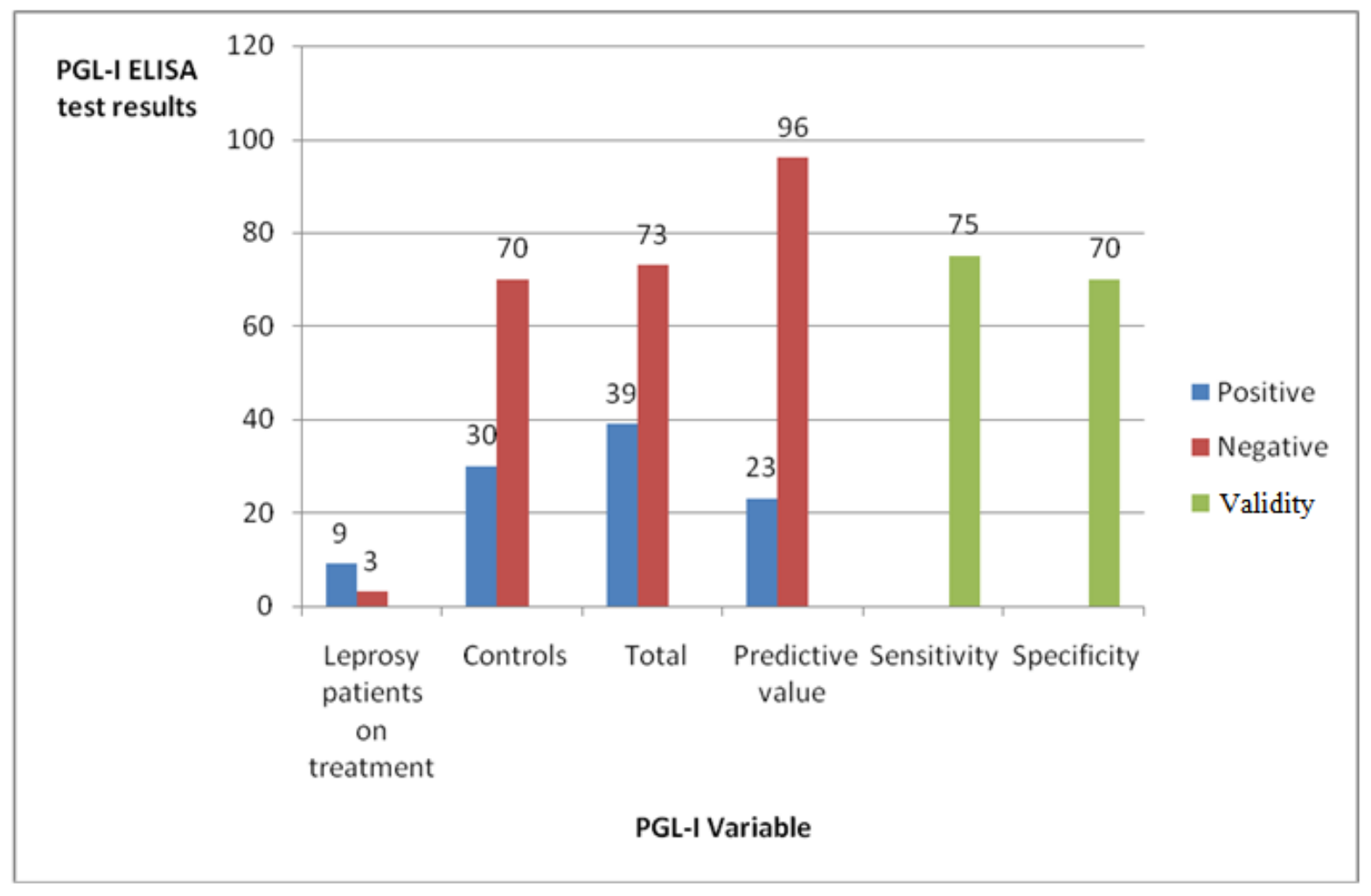

Figure 1. Validity of the PGL-I ELISA for anti-bodies to M. leprae among leprosy patients on treatment and controls

The highest mean optical density of positive samples was observed among leprosy patients (0.14 \pm 0.06$)$, followed by contacts $(0.12 \pm 0.05)$ and controls $(0.04 \pm$ 0.04). The proportion of the study sample that was sero- 
positive for IgM anti-bodies to $M$. leprae was 43.5\% (95\% CI: $37.6 \%$ - 49.4\%). This included 32(50.0\%) leprosy patients, 56(52.3\%) contacts and 30(30.0\%) controls $(p=0.00)$. The breakdown of the validity of the modified PGL-1 ELISA among different categories of the study population and controls is shown in Figures 1, 2, 3 and 4. The results shown in Figure 1 indicate that there was a statistically significant difference in validity between leprosy patients on treatment and controls $\left(\mathrm{X}^{2}\right.$ with Yates correction $=9.56, \quad \mathrm{p}<0.05)$. There was equally a statistically significant difference in validity between contacts of leprosy patients and controls $\left(\mathrm{X}^{2}\right.$ with Yates correction $=9.70, \mathrm{p}<0.05$ ) as shown in Figure 2. The results shown in Figure 3 indicate that there was a statistically significant difference in validity between discharged leprosy patients and controls $\left(\mathrm{X}^{2}\right.$ with Yates correction $=30.51, \mathrm{p}<0.05)$. The results presented in Figure 4 indicate that there was a statistically significant difference in validity between all leprosy patients (active and discharged cases) and controls ( $\mathrm{X}^{2}$ with Yates correction $=5.82, \mathrm{p}=0.01$ ).

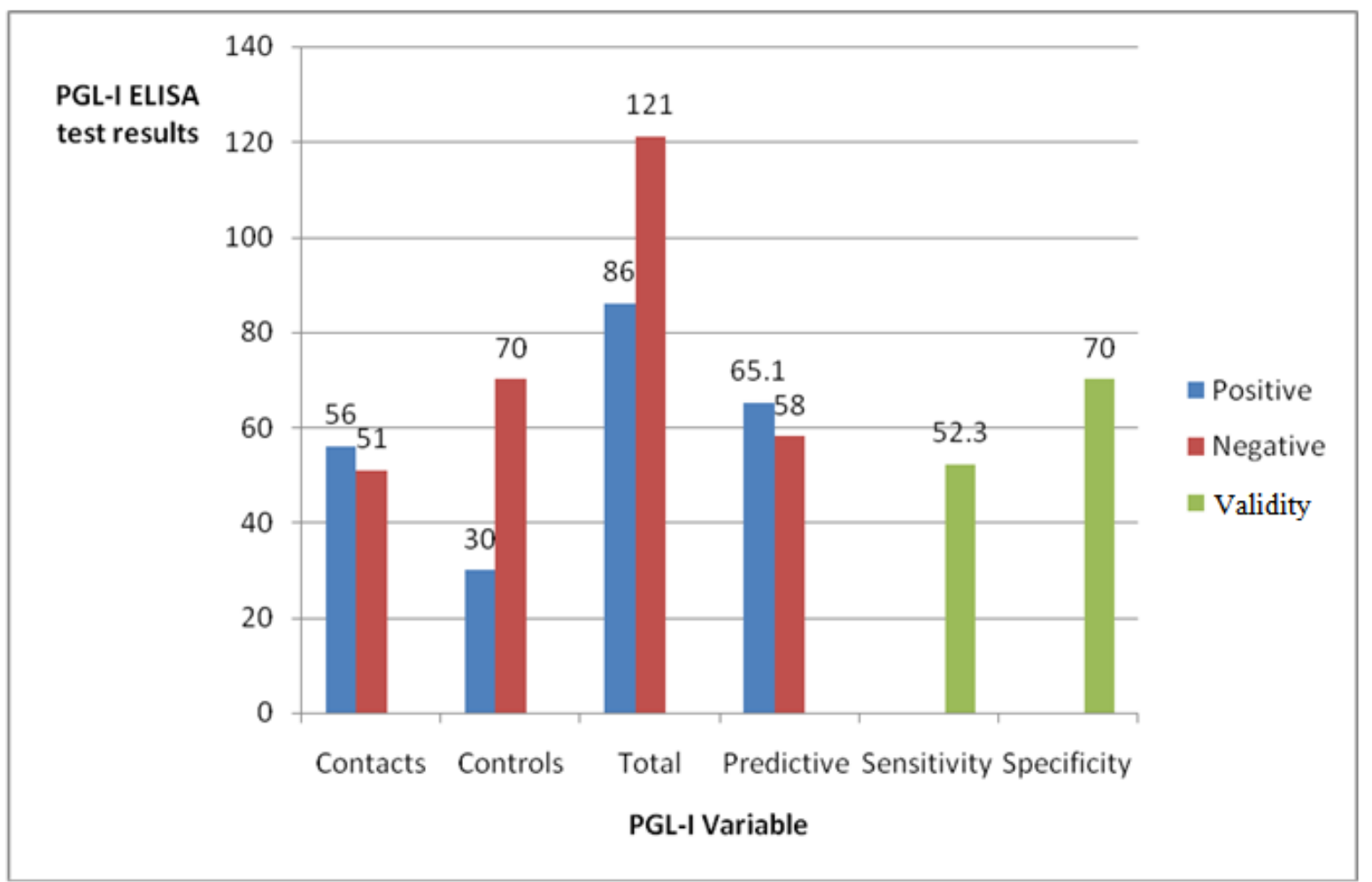

Figure 2. Validity of the PGL-I ELISA for anti-bodies to M. leprae on contacts of Leprosy patients and controls

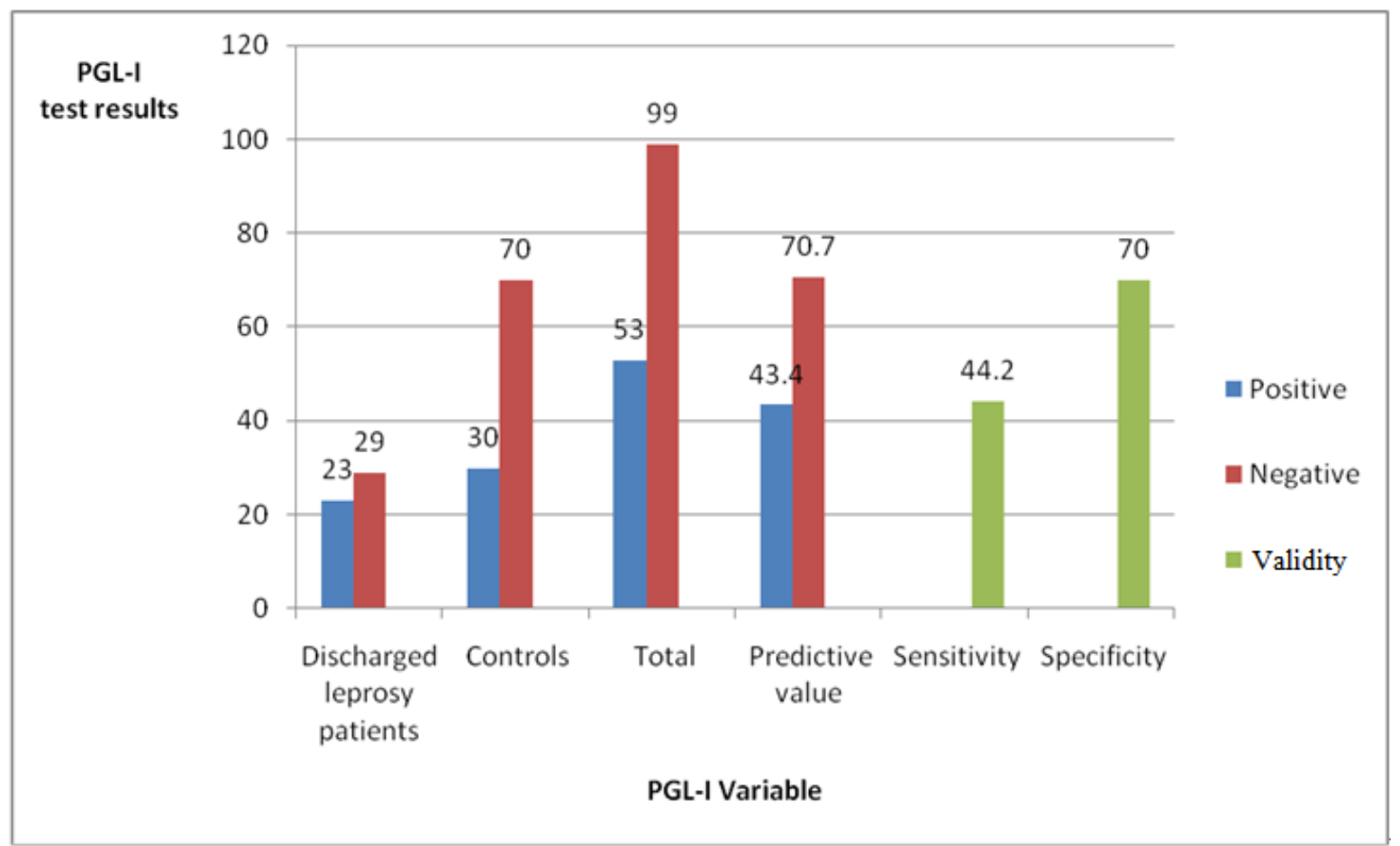

Figure 3. Validity of PLG-I ELISA for anti-bodies to M. leprae on discharged leprosy patients and control 


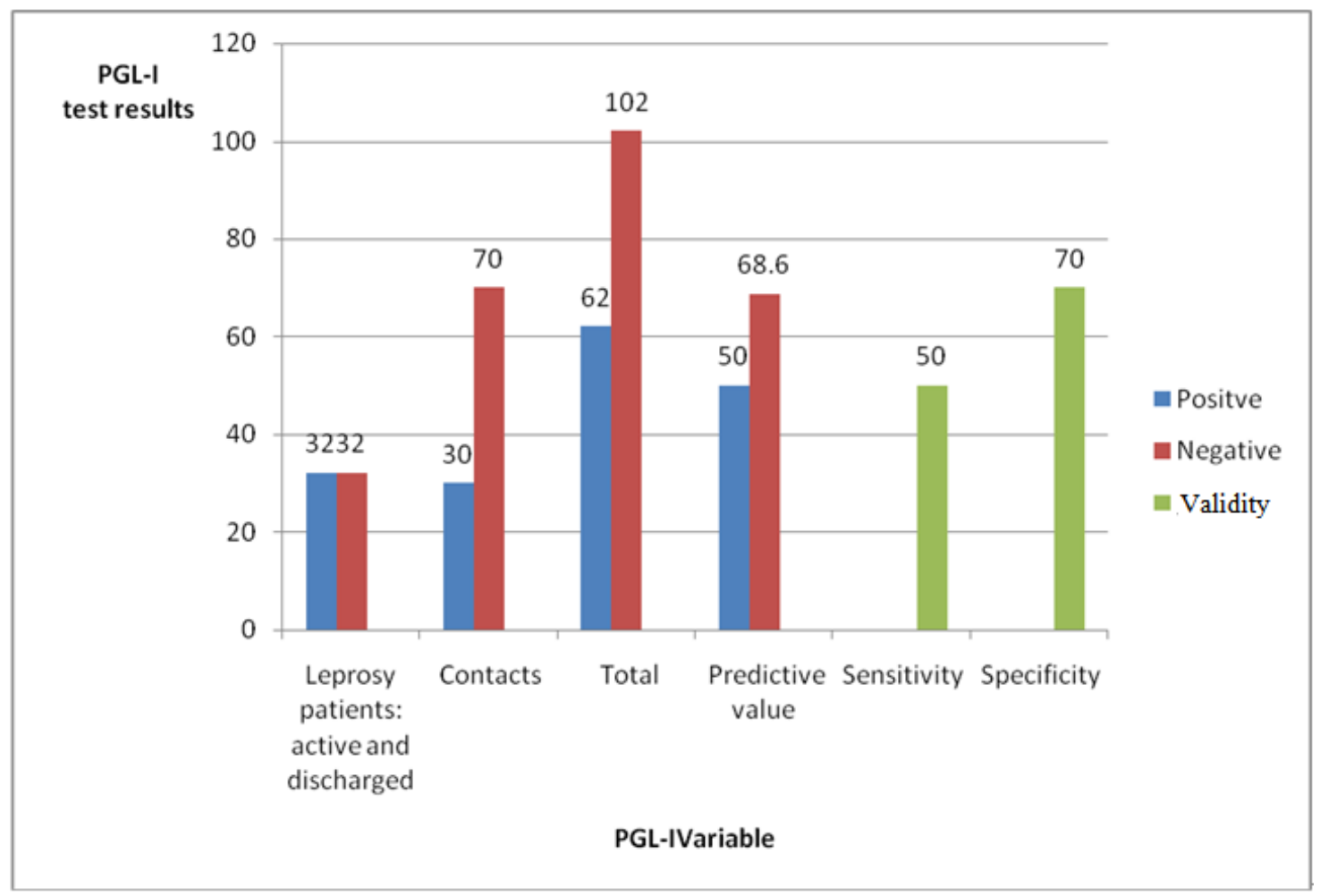

Figure 4. Validity of the PGL-I ELISA for anti-bodies to M. leprae on leprosy patients (active and discharged) and controls

Table 1 shows that there was no statistically significant predictive value of $61.4 \%\left(\mathrm{X}^{2}\right.$ with Yates correction $=0.02$, difference in seropositivity between leprosy patients and the contacts with a specificity of $70 \%$ and negative $\mathrm{p}=0.89)$.

Table 1. Validity of the PGL-I ELISA for anti-bodies to M. leprae on leprosy patients (active and discharged) and contacts

\begin{tabular}{ccccc}
\hline PGL-I ELISA results & Leprosy patients: active and discharged $(\mathbf{n}=\mathbf{6 4})$ & Contacts $(\mathbf{n}=\mathbf{1 0 7})$ & Total (n=171) & Predictive value (\%) \\
\hline Positive & 32 & 56 & 88 & 36.4 \\
Negative & 32 & 51 & 83 & 61.4 \\
Total & $\mathbf{6 4}$ & $\mathbf{1 0 7}$ & $\mathbf{1 7 1}$ & \\
\hline Sensitivity (\%) & & 50 & & \\
Specificity (\%) & & 70 &
\end{tabular}

Table 2. Variation of seropositivity of leprosy IgM antibodies with the socio-demographic and clinical characteristics of the study population

\begin{tabular}{|c|c|c|c|c|}
\hline Socio-demographic characteristics & $\mathrm{n}=\mathbf{2 7 1}$ & Positive (\%) & $\mathrm{X}^{2}$ & P-value \\
\hline \multicolumn{5}{|l|}{ Age group (years) } \\
\hline$<20$ & 91 & 44 & & \\
\hline$\geq 20+$ & 180 & 43 & 0.04 & 0.84 \\
\hline \multicolumn{5}{|l|}{ Gender } \\
\hline Male & 129 & 47 & & \\
\hline Female & 142 & 40 & 0.11 & 0.74 \\
\hline \multicolumn{5}{|l|}{ Marital Status } \\
\hline Single & 115 & 47 & 1.64 & 0.44 \\
\hline Married & 156 & 41 & & \\
\hline \multicolumn{5}{|l|}{ Educational status } \\
\hline None & 51 & 43 & & \\
\hline Primary & 126 & 48 & 1.91 & 0.38 \\
\hline Secondary or up & 94 & 38 & & \\
\hline \multicolumn{5}{|l|}{ Occupation } \\
\hline Farming & 87 & 43 & & \\
\hline Student & 87 & 45 & & \\
\hline Unemployed & 10 & 60 & 0.19 & 0.01 \\
\hline Paid Employment & 40 & 38 & & \\
\hline Self-employed & 47 & 45 & & \\
\hline \multicolumn{5}{|l|}{ Religion } \\
\hline Christian & 257 & 44 & & \\
\hline Muslim & 5 & 40 & 0.93 & 0.82 \\
\hline Others & 9 & 44 & & \\
\hline \multicolumn{5}{|l|}{ Clinical characteristics } \\
\hline \multicolumn{5}{|l|}{ BCG Vaccination Status } \\
\hline BCG Vaccination & 144 & 41 & & \\
\hline No BCG Vaccination & 127 & 46 & 0.08 & 0.74 \\
\hline
\end{tabular}


The proportion of leprosy patients that was seropositive for IgM anti-bodies was 50.0\% ( 95\% CI: 37.8\% $62.3 \%)$. We found that $37.5 \%(n=8)$ pauci-bacillary versus $51.8 \%(n=56)$ multi-bacillary patients were positive for leprosy IgM using the modified ELISA $(\mathrm{p}=0.35)$. Thirty four (43.6\%) intra-familial and 22(76.0\%) extra-familial contacts were positive for anti-bodies to $M$. leprae using the modified PG-1 ELISA $(\mathrm{P}=0.00)$. Out of these, $12(11.2 \%)$ were exposed to pauci-bacillary leprosy and 95(88.8\%) to multi-bacillary leprosy. Table 2 shows the distribution of seropositivity to leprosy IgM antibodies with demographic and clinical characteristics of the study population.

There was a significant difference in sero-positivity of anti-bodies to $M$. leprae in Boyo and Mezam divisions; Boyo division had a higher proportion (51.5\%) compared to $(30.0 \%)$ for Mezam division $(\mathrm{P}<0.05)$.

\section{Discussion}

This sero-epidemiological study was carried out to evaluate the usefulness of a modified PGL-1 ELISA with tris-HCl buffer and IgM globulins as an indicator of subclinical leprosy infection and to monitor leprosy chemotherapy, by analysing serum samples obtained from Boyo and Mezam divisions of Cameroon with high and low leprosy prevalence respectively. The discovery and the elucidation of the chemical structure of a specific phenoglycolipid of M. leprae [17] and the findings that it was antigenic [18] was a major breakthrough in leprosy research. It was thought that the serological method using the PGL-1ELISA with tris-HCl as buffer instead of the traditional phosphate buffer saline (PBS) would be useful in identifying among close contacts of leprosy patients those infected by the bacterium and to monitor chemotherapy $[17,18]$. The IgM class has been reported to be the specific marker of leprosy infection [19] hence this sero-epidemiological study was carried out to evaluate the usefulness of this modified PGL-1 ELISA by collecting and analysing serum samples from leprosy patients, their contacts and a control group. This study has proven the usefulness of the PGL-1 ELISA as a sero-epidemiological tool with D-BSA as antigen and tris- $\mathrm{HCl}$ as buffer for the detection of sub-clinical leprosy infection and the monitoring of chemotherapy. The sensitivity of $75 \%$ reported in this study clearly indicated that the PGL-1 ELISA holds promise as a good marker of leprosy subclinical infection for epidemiological purposes. The results indicate that the determination of anti-PGL-1 antibody levels in an ELISA using tris-HCl as buffer instead of PBS is not only a suitable method for the detection of leprosy subclinical infection but is also a sensitive tool for the evaluation of leprosy chemotherapy.

The use of tris- $\mathrm{HCl}$ as buffer [10], in Paraguay resulted in a sensitivity of the PGL-1 ELISA of $53.1 \%$ in PB and 98.0\% in MB patients. The Paraguan study differs from the current study on a number of aspects. First, StichtGroh's group [10] worked exclusively on active patients while we worked on both active and discharged patients. Furthermore, PGL-1 levels are supposed to decrease since some patients were on treatment; therefore, in the present study, levels of PGL-1 in the mixed group of patients were expected to be lower. We used a larger sample size to ascertain the true PGL-1 level in the active patient group. Secondly, Sticht-Groh's group [10] did not use any contact subjects who are high-risk groups and therefore of public health importance. Thirdly, the prevalence of leprosy, sanitary conditions, socio-economic development, sample size and the genetic background of the subjects are technical factors that could be responsible for the differences between the two studies.

\subsection{Cut-off Points and Predictive Values of the PGL-1 ELISA}

The cut-off points in this study varied from 0.14 to 0.04 which are different from what was found in Cuba [20] and India [21] in which cut off points were 0.100 and 0.07 respectively. Since there is no "gold standard" for the detection of sub-clinical leprosy infection using the PGL-1 ELISA technique, the PGL-1 ELISA with tris- $\mathrm{HCl}$ as a buffer is a more sensitive test than the PGL-1 ELISA with phosphate buffered saline (PBS) as buffer [10]. Sinha and colleagues [22] also examined different levels of cut offs, but none was found to be sufficiently sensitive and specific. Since the PGL-1 ELISA serological assay using tris- $\mathrm{HCl}$ as buffer provide evidence of past or present infection and monitor of chemotherapy, it can provide additional information on patterns of $M$. leprae infection, besides clinical observations.

\subsection{Optical Density among the Leprosy Patients, Their Contacts and Controls}

Using different cut-off values for positivity, consistent patterns were observed in the two divisions among leprosy patients, contacts and control subjects, suggesting that IgM was specifically bound to the D-BSA antigen. There was a significant association in the prevalence of leprosy with anti-body levels among the contact and control subjects. There is an increased risk for leprosy in household contacts of leprosy patients compared with non-contacts $[23,24]$. In our current study, contrary to expectations, $30.0 \%$ of the controls were sero-positive for leprosy IgM anti-bodies which sharply contrasts the findings of the Paraguan study which reported no seropositive cases among the 159 control subjects [10].

In India, George and collaborators [25] found a risk factor of 2.5 in a case-control study in an exposed group compared to those in an unexposed group. A much higher risk factor of 6 was observed among contact and control subjects in our study suggesting that there is a high level of transmission of leprosy among the contact subjects. The majority of the contact subjects were of a low socioeconomic background, implying poor nutritional status and housing conditions which could enhance the chances of transmission of leprosy within this setting [26].

\subsection{Sero-positivity among Active and Discharged Leprosy Patients}

In this current study, $44.3 \%$ discharged patients were still sero-positive for IgM leprosy anti-bodies in the PGL-I ELISA which could be due to persisting M. leprae. Therefore, the efficacy of MDT must be verified through detection of viable organisms in these individuals. 


\subsection{Geographical Distribution of Leprosy Sero-Positivity in the Study Area}

The higher sero-positivity proportion from Boyo division could be due to the location of the leprosarium in Mbingo from where leprosy patients act as reservoirs of infection, hence maintaining a niche for M. leprae. The population in Boyo may also have a different genetic background to that of Mezam. A difference in seropositivity in the study area independent of the leprosy prevalence could also be due to differences in pathogenicity in strains of $M$. leprae, in spite of the fact that no difference in patterns of pathogenicity has been reported. It has been suggested [27] that $M$. leprae identified by species-specific monoclonal anti-bodies could be found in the soil in several non-endemic areas. In rural African settings, socio-economic status is generally low, hygiene and sanitation are poor and overcrowding is common. These factors need to be considered when interpreting results from these divisions. The high population density in Mezam could imply very intense transmission which may explain why $30.0 \%$ controls in this locality were positive for leprosy anti-bodies.

\subsection{Variation of Leprosy Sero-Positivity with the Socio-Demographic and Clinical Characteristics of the Study Population}

The IgM level has been reported to increase during young age and to decrease subsequently with increasing age [28]. In low endemic areas, the occurrence of the bulk of leprosy cases is among older adults [29]. In our study, the highest absorbance was observed among adults. Therefore, these findings do not reflect the general age trend in immunoglobulin synthesis but can be attributed to the presence of anti-bodies binding specifically to the DBSA antigen [30]. Also, the prevalence of the disease in children reflects high transmission of the disease, which is not expected in a low prevalence area.

Sero-positive subjects were found to be concentrated among farmers and students as leprosy is known to affect people of a low socio-economic status such as peasant farmers [31]. Increased overcrowding among students is a risk factor that can enhance the transmission of leprosy.

The preponderance of males over females having leprosy has been reported from Cameroon before [29] as well as from Yemen [32]. Males in general expose themselves to greater risks of infection because of their life style, for example working bare body to remove sand from rivers or digging toilets [30].

An association of serological data with history of BCG vaccination was found indicating that BCG vaccination affects the antibody responses to $M$. leprae specific antigen. This study contrasts findings of other authors $[33,34]$ in population studies where leprosy patients were vaccinated with BCG. It is also highly likely that the variations are due to immunological effects of exposure to various environmental mycobacterial other than BCG and the pathogenic strains, M. leprae and M. tuberculosis [35].

\subsection{Public Health Importance of the PGL-I ELISA Leprosy Screening Test}

Policy related to contacts and control subjects who tested positive require some caution. These cases need to be followed-up at least annually for clinical examination and development of leprosy symptoms with periodic testing using the modified PGL-1 ELISA. There is effective leprosy treatment so those tested positive, in highly endemic areas, should be treated as a prophylactic measure else they continue to act as asymptomatic transmitters of infection. The discharged leprosy patients who tested positive need also to be re-evaluated for relapses, which is an important measure of leprosy control and elimination.

\section{Conclusion}

Leprosy has been eliminated in Cameroon according to the WHO(less than 1 case per 10,000 population) but there still endemic foci in some localities. The detection of subclinical infection is still a problem as most clinical cases show up with deformities. The PGL-1 ELISA with D-BSA using tris-HCl as buffer is a useful epidemiological surveillance tool for the detection of subclinical leprosy infection and monitoring of chemotherapy because a sensitivity of $75 \%$ was recorded. The $44.3 \%$ discharged leprosy patients who tested positive should be re-evaluated for relapses. A total of $43.6 \%$ intra-familial contacts and $76.0 \%$ extra-familial contacts were positive for antibodies to $M$. leprae. Subsequent studies in leprosy endemic communities should compare Tris- $\mathrm{HCl}$ and PBS as buffers to shade more light on the role of the modified PGL-1 ELISA as an epidemiological surveillance tool.

\section{Competing Interests}

The authors declare that this work has no competing interests.

\section{Acknowledgements}

Dr. U. Fruth of the Vaccine unit of WHO, Geneva, donated the D-BSA; Professor Claude Muller of the WHO Collaborating for Measles in Luxemburg supplied the antihuman IgM peroxidase enzyme for the PGL-I ELISA; Professor Mark Nwogwu of the University of Ibadan provided the substrate and tween-20 for the ELISA and Dr. Sang-Nae Cho of the Yonsei University College of Medicine in Korea provided the methodological recommendations for the reconstitution of the D-BSA and BSA and their use in the ELISA. We are indebted to Mr. David Nfiekwe, Mr. Abel Sande, Mr. Ngam and Dr. R. Zimmermann of the Mbingo leprosarium, Dr. .Jerry Fluth of the Health Services of the Cameroon Baptist Convention; Mr. Fonda Solomon \& Mr. Nfor Cajetan of the Bamenda Regional Hospital for data collection. Miss Omoyeme Henrietta of the Chemical Pathology Department of the College of Medicine of the University of Ibadan offered technical assistance in the ELISA technique. This project was sponsored by Mr. Nsagha BN (Late) and Papa James Nsagha (late) to whom the authors are grateful indeed. 


\section{Author's Contribution}

DSN substantially contributed to the conception and design, acquisition of data, analysis and interpretation of data, drafting the article and revising it critically for important intellectual content; EAB and LS substantially contributed to conception and design, analysis and interpretation of data, drafting the article and revising it critically for important intellectual content; ACZKB and ENB substantially revised the manuscript for important intellectual content; JCNA, ALN, HLFK and NNM substantially revised the manuscript for important intellectual content. AKN contributed to the design, interpretation of the data and substantially revised the manuscript for important intellectual content. All authors read and approved the final version of the manuscript before publication.

\section{References}

[1] Cardona-Castro N, Beltran-Alzate JC, Manrique-Hernandez R (2008) Survey to identify Mycobacterium leprae infected household contacts of patients from prevalent regions of leprosy in Colombia. Mem. Inst.. Oswaldo Cruz. 103:0074-0276.

[2] Meima A, Smith WCS, van Ootmarssen GJ, Richardus JH, Habbema DF (2004) The future incidence of leprosy: a scenario analysis. Bull. World Health Organ. 5: 373-380.

[3] Lockwood DN (2002) Leprosy elimination - a virtual phenomenon or a reality? BMJ. 324: 1516-1518.

[4] Lockwood DNJ, Kumar B (2004) Treatment of leprosy. BMJ. 328: 1447-1448.

[5] Naafs B (2000) Viewpoint: leprosy after the year 2000. Trop. Med. Int. Health. 5: 400-403.

[6] Visschedijk J, van de Broek, Eggens H, Lever P, van Beers S, Klatser P (2000) Mycobacterium leprae-millennium resistant! Leprosy control on the threshold of a new era. Trop. Med. Int. Health. 5: 388-399.

[7] Gupta V, Bhushan K, Dogra S, Vasudev U, Kaur I (2004) Leprosy elimination -How far? Lep. Rev. 75: 101-102.

[8] Nsagha, DS, Bamgboye, EA, Oyediran, ABOO (2009a) Childhood leprosy in Menchum division of Cameroon: Result of a school survey and chart Review. NQJHM; 19: 125-130.

[9] Nsagha, DS, Bamgboye, EA, Oyediran, ABOO (2009b) Operational barriers to the implementation of multidrug therapy and leprosy elimination in Cameroon. Indian J Dermatol Venereol Leprol; 75: 469-75.

[10] Sticht- Groh V, Alvarenga AE, Vetton L, Ballestrem WV (1992) Use of a different buffer system in the phenolic glycolipid-1 ELISA. Int. J. Lepr. 60: 570 -574.

[11] Cho SN, Yanagihara DL, Hunter SW, Gelber RH, Brennan PJ (1983) Serological specificity of phenolic glycolipid I from Mycobacterium leprae and use in serodiagnosis of leprosy. Infect Immun 41: 1077-1083.

[12] Buhrer-Sekula S (2008) PGL-1 leprosy serology. Revista Socicdade Brasilcira Med Trop 41: 3-5.

[13] WHO (2014). Leprosy Elimination. Leprosy Surveillance Systems. Available at: http://www.who.int/lep/monitor/surveillance/en/. [Accessed May 1, 2014].

[14] Moura MLN, Dupnik KM, Sampaio GAA, Nóbrega PFC, Jeronimo AK, et al. (2013) Active Surveillance of Hansen's Disease (Leprosy): Importance for Case Finding among Extradomiciliary Contacts. PLoS Negl Trop Dis 7(3): e2093.
[15] Shen J, Wang Y, Zhou M, Li W (2009) Analysis on value of household contact survey in case detection of leprosy at a low endemic situation in China. Indian J Dermatol Venereol Leprol 75 : 152-6.

[16] Shen JP, Gupte MD, Jiang C, Manickam P, et al. (2005) Trends of case detection and other indicators of leprosy in China during 1985-2002. Chin Med Sci J 20: 77-82.

[17] Hunter SW and Brennan PJ (1981) A novel phenolic glycolipid of Mycobacterium leprae possibly involved in immunogenicity and pathogenicity. J. Bacter. 147: 718-735.

[18] Hunter SW, Fujiwara T and Brennan PJ (1982) Structure and antigenicity of the major specific glycolipid antigen of Mycobacterium leprae. J. Biol Chem. 258: 15072-15078.

[19] Wang D (1997) Serological surveillance with PGL-I and LAM-B for leprosy relapse. Can. Lepr. J. 13: 138-140.

[20] Gonzalez-Abreu E, Mora N, Perez M, Pereira M, Perez J, Gonzalez AB (1990) Serodiagnosis of leprosy in patients' contacts by enzyme-linked immunosorbent assay. Lep. Rev. 61: 145-150.

[21] Patil SA, Katoch K, Ramu G and Sengupta U (1995) Detection of antibodies against phenolic glycolipid-1 (PGL-1), 35-KDa and 30$40-\mathrm{KDa}$ components of Mycobacterium leprae in the cerebrospinal fluid of leprosy patients. J. Med. Microbiol. 43: 115119.

[22] Sinha S, Kannan S, Nagaraju B, Sengupta U and Gupte MD (2004) Utility of serodiagnostic tests for leprosy: a study in an endemic population in South India. Lepr. Rev. 75: 266-273.

[23] Noordeen SK (1978) Infectivity of leprosy. In : Chatterjee, BR (ed). A window on leprosy. Ghandi Memorial Leprosy Foundation. New Delhi; pp 59-63.

[24] Fine PEM, Sterne JAC, Ponnighaus JM,Bliss L,Saul J, Chihana A et al (1988a) Household and dwelling contact as risk factors for leprosy in Northern Malawi. Am. J. Epid. 56: 243-254.

[25] George K, John KR, Muliyil JP, Joseph A (1990) The role of intrahousehold contacts in the transmission of leprosy. Lep. Rev. 61: 60-63.

[26] Ong AKY, Frankkei RI, Maruyama MH (1999) Cluster of leprosy cases in Kona, Hawaii: Impact of the compact of free association. Int. J. lepr. 67: 13-18.

[27] Kazda J, Irgens M, Kolk AHJ(1990) Acid fast bacilli found in sphagnum vegetation of coastal Norway containing Mycobacterium leprae specific phenolic glycolipid 1. Int. J. Lepr. 58: 353-357.

[28] Buckley CE, Dorsey FC (1970) The effect of ageing on human serum immunoglobulin concentration. J. Immunol. 105: 964-872.

[29] Noordeen SK (1985) The epidemiology of leprosy. In: Leprosy, Hastings,RC (ed).Churchill Livingstone, Produced by Longman Group Ltd.Hong Kong. pp 15-30.

[30] Nsagha DS (2002) Epidemiology and community perception of leprosy in the Boyo, Menchum and Mezam divisions of Cameroon. PhD thesis, University of Ibadan, Ibadan, Nigeria. Pages 1-394.

[31] Ponnighaus JM, Fine PEM, Sterne JAC, Malema SS, Bliss L, Wilson RJ (1994) Extended schooling and good housing conditions are associated with reduced risk of leprosy in rural Malawi. Int. J. Lepr. 62: 345-352.

[32] Al-Qubati Y, Al-Dobai BM (1999) Review of leprosy control in Yemen. Int. J. Lepr. 67: 150-153.

[33] Fine PEM, Ponnighaus JM, Burgess P, Clarkson JA, Draper CCM (1988b) Seroepidemiological studies of leprosy in Northern Malawi based on an ELISA using synthetic glycoconjugate antigen. Int. J. Lepr. 56: 243-254.

[34] Douglas JT, Hirsch DS, Fajardo TT, Guido LS, Klatser PR (1990) Serological monitoring of previously treated lepromatous patients during a course of multiple immunotherapy treatments with heatkilled Mycobacterium leprae and BCG. Clin Exper. Immunol. 82: 563-573.

[35] Fine PEM (1995) Variations in protection by BCG implications of and for heterologous immunity. Lancet. 346: 1339-1345. 